\title{
Dominant dystrophic epidermolysis bullosa, nails only
}

INSERM

\section{Source}

INSERM. (1999). Orphanet: an online rare disease and orphan drug data base. Dominant dystrophic epidermolysis bullosa, nails only. ORPHA:158676

Dystrophic epidermolysis bullosa, nails only is a rare subtype of dystrophic epidermolysis bullosa (DEB, see this term) that shows no blistering and that is characterized by dystrophic or absent nails. 